\title{
Acordos de compartilhamento de risco entre sistema públicos de saúde e a indústria farmacêutica de medicamentos
}

Gabriela Araujo VALENCIA: Faculdade de Saúde Pública - FSP, Universidade de São Paulo - USP. ORCID: https://orcid.org/0000-0002-5537-163X

Jaqueline Vilela BULGARELI: Faculdade de Odontologia - FO, Universidade de Uberlândia - UFU. ORCID: http://orcid.org/0000-0002-5632-4333

\section{Resumo}

O envelhecimento da população e aumento da prevalência de doenças crônicas são uma realidade que desafiam e desafiarão os Sistemas Públicos de Saúde, uma vez que aumentam significativamente os gastos com medicamentos. Por sua vez, as indústrias farmacêuticas lançam medicamentos cada vez mais rápido, caros e específicos. A incorporação de novos medicamentos é uma etapa frágil para o gestor público que fica responsável por todos os riscos caso o medicamento não funcione ou quanto ao tamanho exato da população que irá usar. A fim de garantir o acesso à população e diminuir o risco do pagador na etapa de incorporação e compra dos medicamentos, desde os anos 2000, são propostos os chamados acordos de compartilhamento de riscos (risk sharing agreement entre outras denominações). Acordos de compartilhamento de risco podem ser baseados em critérios financeiros como volume e desconto e, os mais recentes, baseados em performance. Neste último cenário, o pagador, no caso o Sistema Público de Saúde, aceita incorporar por um certo período pré-determinado uma droga para um grupo de pacientes específicos na condição da Indústria Farmacêutica aceitar um preço dependente do desfecho real que a droga apresentar. Dessa forma, o risco antes concentrado no gestor, passa a ser dividido com a Indústria Farmacêutica. Com esse tipo de acordo, o pagador também visa uma futura flexibilização do preço do medicamento. Fica evidente que acordos de compartilhamento de risco são bem-vindos para o caso de medicamentos onerosos e que nem sempre possuem completa evidência do desfecho clínico como drogas oncológicas, terapias gênicas e para doenças raras. Além da incontestável barreira da capacidade de negociação do gestor, acordos de compartilhamento de risco pressupõem

Recebid o: 1 out. 2020

Aceito: 21 out. 2020

Autor de correspondência: Gabriela Araujo Valencia gabivalenciagabi@gmail.com

Conflito de interesses: As autoras declaram não haver nenhum interesse profissional ou pessoal que possa gerar conflito de interesses em relação a este manuscrito.

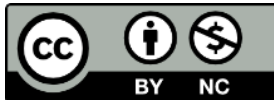
a necessidade de coleta de dados de qualidade da vida real, pois em muitos casos o desfecho do paciente é condição primária para o pagamento da terapia. Esbarra-se em outro grande desafio: definir qual o indicador de desfecho clínico será avaliado. Soma-se a necessidade de sistema de informação com capacidade de concentrar dados desse indicador, questões de confidencialidade, transparência e burocracia além da definição de quem fará a avaliação desse indicador. Em paralelo, deve-se definir o tempo que esse monitoramento irá ocorrer. É notável que haja preocupação importante da perspectiva jurídica e de gestão desses acordos de compartilhamento de risco. Outros desafios podem ser destacados no campo econômico. Por exemplo, relata-se na literatura a falta de informações financeiras pós implementação, para quantificar se os resultados realmente foram alcançados. Mesmo com essa falta de dados em geral, há exemplos quantitativos favoráveis. Visto que o SUS está iniciando a realização de acordos inovadores, neste presente trabalho, objetivou-se avaliar o que a literatura científica traz sobre as condições que viabilizam a implementação de acordos de compartilhamento de risco entre Sistema Públicos de Saúde e a indústria farmacêutica de medicamento. O método proposto é uma revisão integrativa da literatura nas bases de dados BVS e PubMed. Para tal determinou-se 
a seguinte pergunta de pesquisa: "O que a literatura científica apresenta sobre acordos de compartilhamento de risco entre os Sistemas Públicos de Saúde e a indústria farmacêutica de medicamentos?". A estratégia de busca foi dividida entre descritores em português, segundo DeCS, para busca na base BVS e descritores conforme MeSH, em inglês, para busca no PubMed. A sintaxe final foram: na BVS ((mh:("Participação no Risco Financeiro")) OR (mh:("Reembolso de Incentivo")) OR (mh:("Avaliação de Resultados em Cuidados de Saúde")) OR (mh:("Mecanismo de Reembolso"))) AND ((mh:("Medicina Estatal")) OR (mh:("Assistência de Saúde Universal")) OR (mh:("Programas Nacionais de Saúde")) OR (mh:("Política de Saúde")) OR (mh:("Sistema Único de Saúde")) OR (mh:("Cobertura Universal do Seguro de Saúde")) OR (mh:("Sistemas de Saúde")) OR (mh:("Programas Governamentais")) OR (mh:("Cobertura Universal de Saúde")) OR (mh:("Acesso Universal aos Serviços de Saúde")) OR (mh:("Estratégias para Cobertura Universal de Saúde"))) AND ((mh:("Preparações Farmacêuticas")) OR (mh:("indústria farmacêutica")) OR (mh:("custos de medicamentos"))) e no Pubmed, ((("Reimbursement Mechanisms"[MeSH Terms] OR "outcome assessment, health care"[MeSH Terms]) OR "reimbursement, incentive"[MeSH Terms]) OR "risk sharing, financial"[MeSH Terms]) AND ((((("State Medicine"[MeSH Terms] OR "Public Health Administration"[MeSH Terms]) OR "Universal Health Care"[MeSH Terms]) OR "National Health Programs"[MeSH Terms]) OR "Health Policy"[MeSH Terms]) OR "Universal Health Insurance"[MeSH Terms]) OR "Government Programs"[MeSH Terms])) AND ("Pharmaceutical Preparations"[MeSH Terms] OR "Drug Industry"[MeSH Terms]) OR "Drug Costs"[MeSH Terms]). Dos 722 artigos levantados, a análise decorreu de 22 artigos, após exclusões por idioma, duplicata e aproximação com o tema. Como resultados esperados, pretende-se, a partir da experiência em outros países, conhecer os tipos de acordos de compartilhamento de risco e examinar as condições econômicas, jurídicas e/ou de gestão que limitaram ou promoveram esses acordos. Tem-se que acordos de compartilhamento de risco são recentes, portanto, ainda não há uma metodologia padrão de como devem ou não serem implementados, entretanto são extremamente contemporâneos e em evolução. Em análise preliminar dos resultados da estratégia de busca, observou-se que os artigos se concentram nos anos de nos anos mais recentes, especialmente após 2010, evidenciando que os acordos estão aumentando em número. A grande maioria trata de experiências relacionadas a medicamentos oncológicos ou para doenças raras, por serem onerosos e muitas vezes com baixa quantidade de evidências clínicas. É observado que os artigos se concentram em países europeus e que a discussão, definição e implementação dos acordos estão intimamente relacionadas ao processo de Avaliação de Tecnologia em Saúde. Os autores mostram-se otimistas com os acordos de compartilhamento implementados como forma alternativa de compra, mencionando que o acesso ao medicamento pela população foi facilitado e houve significativa diminuição dos gastos pelos Sistemas de Saúde. No entanto, levantam barreiras importantes pósimplementação, como dificuldade de coleta de dados e poucos estudos que quantifiquem o impacto financeiro. Muitos artigos citam superficialmente os critérios, condições jurídicas e de gestão em qual os acordos de compartilhamento de risco foram celebrados. Tal fato pode indicar que, embora haja ganho evidente na celebração de acordos de compartilhamento de risco, há necessidade de que todas as partes envolvidas se aprofundem na discussão para que possam definir, por exemplo, diretrizes para expansão dessa modalidade.

Descritores: Participação no Risco Financeiro; Política de Saúde; Medicina Estatal; Custos de Medicamentos; Indústria Farmacêutica.

Keywords: Risk Sharing, Financial; Health Policy; State Medicine; Drug Costs; Drug Industry.

Palabras clave: Prorrateo de Riesgo Financiero; Política de Salud; Medicina Estatal; Costos de los Medicamentos; Industria Farmacéutica. 